\title{
Elective use of left ventricular assist after delayed arterial switch operation for D-transposition of the great vessels with intact ventricular septum
}

\author{
James D. St. Louis, MD, ${ }^{a}$ Derek Wheeler, MD, ${ }^{b}$ and Henry B. Wiles, MD, ${ }^{a}$ Augusta, Ga, and Cincinnati, Ohio
}

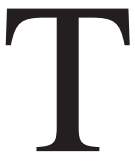
he arterial switch operation (ASO) has become the procedure of choice for D-transposition of the great arteries with intact ventricular septum (DTGA-IVS).

Operative outcomes are improved when repair is undertaken during the neonatal period. ${ }^{1}$ Late presentation or elective delay in operative correction presents a therapeutic dilemma. "Deconditioning" of the myocardium can occur after exposure to a lowpressure circulation. The method by which the ventricle might be "reconditioned" remains controversial. We report our experience with an 8-month-old in whom a neonatal ASO was contraindicated. The child underwent an uncomplicated ASO followed by 4 days of left ventricular support.

\section{Clinical Summary}

The patient for presentation is an 8-month-old boy who had presented as a newborn with the diagnosis of primary pulmonary hypertension. On admission, the patient was in profound shock, with arterial saturations of $40 \%$. Echocardiography revealed the diagnosis of DTGA-IVS with a severely restrictive patent foramen ovale. A Rashkind balloon septostomy was successfully accomplished. Head ultrasonography revealed a grade III intraventricular hemorrhage that precluded heparinization. The patient subsequently underwent ligation of the patent ductus arteriosus and was discharged to home at 25 days of life.

Echocardiography performed at 24 days revealed a conicalshaped left ventricle (LV) with a left ventricular end-diastolic diameter (LVEDd) of $1.56 \mathrm{~cm}$. The indexed left ventricular mass (iLVm) was calculated at $49.24 \mathrm{~g} / \mathrm{m}^{2}$. Echocardiography performed at 2 months of age was significant for an LVEDd of 1.12 $\mathrm{cm}$ and an iLVm of $33.96 \mathrm{~g} / \mathrm{m}^{2}$. At 8 months, the LVEDd was 1.14 $\mathrm{cm}$, and the $\mathrm{iLVm}$ was $16.59 \mathrm{~g} / \mathrm{m}^{2}$. There was a significant leftward shift of the ventricular septum (Figure 1).

The patient was taken to the operating room at 8 months of age for an ASO with a planned period of left ventricular support. The

From the Children's Medical Center at the Medical College of Georgia, ${ }^{a}$ Augusta, Ga; and the Cincinnati Children's Hospital Medical Center, Cincinnati, Ohio.

Received for publication March 16, 2007; accepted for publication March 26, 2007.

Address for reprints: James D. St. Louis, MD, Medical College of Georgia, 1120 15th St, BA-4300, Augusta, GA 30912-4040 (E-mail: jstlouis@ mcg.edu).

J Thorac Cardiovasc Surg 2007; 134:252-3

$0022-5223 / \$ 32.00$

Copyright @ 2007 by The American Association for Thoracic Surgery doi:10.1016/j.jtcvs.2007.03.014

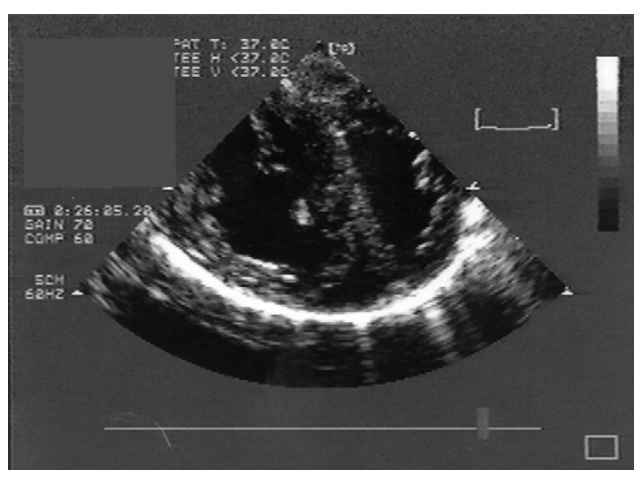

Figure 1. Echocardiograph before the arterial switch operation. Leftward shift of ventricular septum is shown.

diagnosis was confirmed as DTGA-IVS with a single coronary artery from the posterior-facing sinus (Leiden: 2R, AD, Cx). An uncomplicated ASO was accomplished by using a pericardial patch to augment the coronary implantation. The patient was converted from cardiopulmonary bypass to a left ventricular assist device (Bio-Medicus centrifugal pump). Flows were maintained at $100 \mathrm{~mL} \cdot \mathrm{kg}^{-1} \cdot \mathrm{min}^{-1}$ to maintain a pulsatile systemic pressure of 60 to $80 / 40$ to $60 \mathrm{~mm} \mathrm{Hg}$ and a left atrial pressure of 8 to $10 \mathrm{~mm} \mathrm{Hg}$.

Postoperative echocardiography revealed a dramatic change in the geometry of the LV. The ventricular septum shifted to the right, with the left ventricular shape becoming conical (Figure 2). The immediate postoperative LVEDd and iLVm values were similar to preoperative measurements $\left(1.19 \mathrm{~cm}\right.$ and $21.89 \mathrm{~g} / \mathrm{m}^{2}$, respectively). After 4 days of partial left ventricular support, the LVEDd increased to $2.52 \mathrm{~cm}$, with a calculated iLVm of 43.9 $\mathrm{g} / \mathrm{m}^{2}$. At 1 month, the LVEDd diameter was $2.45 \mathrm{~cm}$, and the iLVm increased to $74.3 \mathrm{~g} / \mathrm{m}^{2}$. One year after the ASO, the patient is doing well, with mild dilation of the neoaortic root.

\section{Discussion}

Since being introduced by Jatene in 1985, the ASO has become the procedure of choice for DTGA-IVS. The procedure is undertaken within 3 weeks after birth, before the deconditioning of the LV. Delay in presentation or conditions that preclude early operative intervention present a therapeutic challenge.

Echocardiography has proved valuable in evaluating the deconditioned LV and need for a reconditioning procedure. Left ventricular mass can be estimated by measuring end-diastolic diameter, posterior wall thickness, and interventicular thickness. ${ }^{2}$ An iLVm of $35 \mathrm{~g} / \mathrm{m}^{2}$ or less might indicate the need for LV retaining. ${ }^{3}$

Several investigators have advocated a rapid 2-stage approach in preparing the deconditioned LV for systemic work. ${ }^{4}$ 


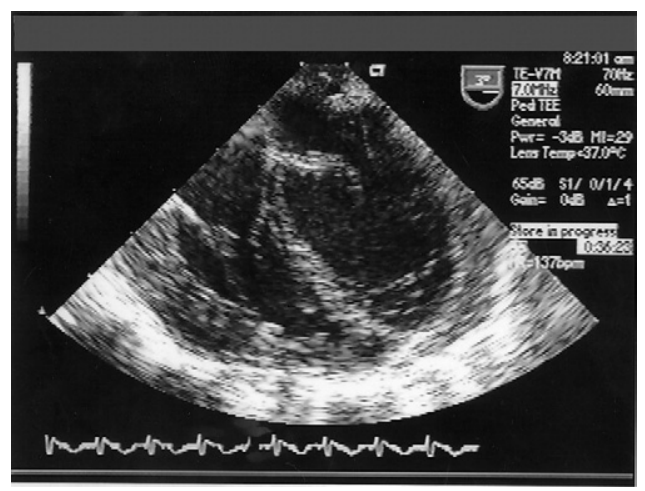

Figure 2. Postoperative echocardiograph after removal from left ventricular assist device.

The procedure uses a tight pulmonary artery band and a systemicpulmonary artery shunt. The postoperative course is associated with significant morbidity. Short-term extracorporeal membrane oxygenation support after ASO has been advocated as an effective mechanism of support while the LV undergoes a period of reconditioning. ${ }^{5}$ We used a simple circuit without an oxygenator to support the systemic circulation during a brief period of myocardial reconditioning. This eliminated many of the complications associated with extracorporeal membrane oxygenation support.
We were able to increase left ventricular afterload and wall stress while maintaining cardiac output in a controlled fashion. A relatively short period of increased wall stress resulted in a dramatic increase in left ventricular mass and the ability to support a systemic work load.

In conclusion, we present the effective use of isolated left ventricular support during a period of myocardial reconditioning after a delayed ASO. The degree of myocardial hypertrophy was sufficient to allow a reasonable period of support without the complications routinely seen with the use of mechanical assist devices. This case supports the notion that postoperative myocardial assistance can be applied not simply as a rescue modality.

\section{References}

1. Wernovsky G, Mayer J, Jonas R, et al. Factors influencing early and late outcomes of the arterial switch operation for transposition of the great arteries. J Thorac Cardiovasc Surg. 1995;109:289-302.

2. Devereux R, Alonso D, Lutas E, et al. Echocardiography assessment of LV hypertrophy: comparison to necropsy findings. Am J Cardiol. 1986; 57:450-8.

3. Gayet-Lacour F, Piot D, Zoghbi J, et al. Surgical management and indication of the left ventricular retraining in arterial switch for transposition of the great arteries with intact ventricular septum. Eur J Cardiothorac Surg. 2001;20:824-9.

4. Jonas R, Giglia T, Sanders S, et al. Rapid two-stage arterial switch for transposition of the great arteries and intact ventricular septum beyond the neonatal period. Circulation. 1989;80(suppl 1):203-8.

5. Mee R, Harada Y. Retraining of the left ventricle with a left ventricular assist device (Bio-Medicus) after the arterial switch operation. J Thorac Cardiovasc Surg. 1991;101:171-3. 